\title{
El estereotipo de género como nuevo valor noticia en los periódicos digitales en Europa ${ }^{1}$
}

\author{
Santiago GALlUR SANTORUM \\ Universidad Autónoma de Ciudad Juárez (Chihuahua, México) \\ santiagostgsstgs@hotmail.com \\ Berta GARCÍA OROSA \\ Universidad de Santiago de Compostela \\ bertago@gmail.com
}

Recibido: 16 de julio de 2014

Aceptado: 4 de diciembre de 2015

\section{Resumen}

El objetivo principal de la investigación es el análisis de los estereotipos de género como nuevo valor noticia en los diarios digitales The Times, El País, Le Monde, Diario de Noticias y Corriere della Sera. La metodología utilizada fue el análisis de contenido de 1688 noticias publicadas entre el 1 de mayo del 2013 y el 1 de mayo del 2014. Los resultados indican que perviven los estereotipos tradicionales de las mujeres especialmente en el caso de El País, El Corriere della Sera y el Jornal de Noticias. Sin embargo, al mismo tiempo aparecen los que denominamos "contra-estereotipos" como un nuevo valor noticia caracterizados por presentar a la mujer con valores positivos opuestos a los estereotipos tradicionales especialmente en The Times y Le Monde.

Palabras clave: periodismo digital, perspectiva de género, producción, estereotipos, medios de comunicación.

\section{Gender stereotyping as the new value in the electronic newspapers in Europe}

\begin{abstract}
The main goal of the research is the analysis of gender stereotypes as a new news value in digital media The Times, El País, Le Monde, Journal de Noticias and Corriere della Sera. The methodology used was content analysis of 1688 news published between May 1, 2013 and May 1, 2014. Results indicate the presence of the traditional stereotypes of women especially in the case of The Times, El Pais, Corriere della Sera and the Jornal de Noticias. However, at the same time it is shown what we call "counterstereotypes" as a new news value characterized by presenting women with positive ideas opposed to traditional stereotypes especially in The Times and Le Monde.
\end{abstract}

Keywords: digital journalism, gender perspective, newsmaking, stereotype, media.

\section{Referencia normalizada}

GALLUR SANTORUM, Santiago y GARCÍA OROSA, Berta (2016): "El estereotipo de género como nuevo valor noticia en los periódicos digitales en Europa". Estudios sobre el Mensaje Periodístico. Vol. 22, Núm. 1 (enero-junio), págs.: 287-298. Madrid, Ediciones Complutense.

Sumario: 1. Introducción y marco teórico. 2. Metodología. 3. Análisis de datos. 4. Conclusiones. 5. Referencias bibliográficas.

1 Este artículo es producto del Proyecto del Ministerio de Economía y Competitividad (referencia: CSO2012-38467-C03-03) y Apoyo a Incorporación de nuevos PTC en el marco del programa de Mejoramiento de Profesorado (PROMEP), México. 


\section{Introducción y marco teórico}

La investigación se enmarca dentro de los estudios de género en comunicación que, desde un principio, desvelaron las implicaciones ideológicas de los distintos conceptos de género que empleaban los discursos sociales con el fin de tomar conciencia de la violencia simbólica que significaban concepciones de la mujer como "ángel del hogar" y de la negación de la mujer como sujeto social. En última instancia, se describía cómo a las mujeres se les negaba la posibilidad de narrarse a sí mismas (Kivikuru et al, 1999).

En función de las teorías de la producción de la información (Tuchman, 1983; Fishman, 1980) y, bajo la perspectiva que explica la información periodística como una construcción de la realidad, surge también otro grupo de investigaciones que intentan ver la presencia e importancia de la mujer en el proceso productivo de los medios de comunicación (Gallego, 2002) cuando los investigadores destapan como insuficiente el análisis de los discursos ya elaborados y comprenden la importancia de analizar todas las fases del proceso productivo desde la perspectiva de género.

Las conclusiones de las investigaciones emprendidas en los últimos decenios del siglo XX han comprendido a los medios como constructores de la realidad y, por lo tanto, como los instrumentos fundamentales para la transmisión de valores estereotipados y patriarcales sobre las mujeres y los valores femeninos. Un discurso mediático que se construye de forma hegemónica, aunque, desde la teoría feminista, es que la "clase dirigente" está atravesada por el género como elemento estratificador que la define.

Pese a que la presencia de la mujer en los medios de comunicación ha aumentado, todavía es menor a la de los hombres y tienen escasa visibilidad en portada (Marín, Armentia, Caminos, 2011; Aladro, Padilla, Requeijo, Semova, García, García, Viñarás, 2014; Gómez y Patiño, 2011). La figura de la mujer continúa mayoritariamente relegada a secciones blandas de los medios (Gallego, 2013; Marín, Ganzabal, 2011) y, en general, su imagen continúa siendo estereotipada y con un enfoque reducido a los roles tradicionales (Tous, Meso, Simelio, 2013).

Pese a que en la década de los 90 surgieron algunos estudios que revelaban ciertas transformaciones en los estereotipos de género y apuntaban un tímido descenso en la representación de las mujeres como elemento decorativo y una diversificación de sus ocupaciones (García, Martínez, 2009), los últimos trabajos realizados sobre medios digitales continúan indicando una tendencia realmente negativa (Franquet, Luzón, Ramajo, 2007; Mateos de Cabo; Gimeno Nogués; Martínez Martínez, 2009).

En esta línea, esta investigación propone el análisis de los estereotipos tradicionales como valores noticia y en el trascurso de la misma desvela la construcción de los que se denominaron "contra-estereotipos" como un nuevo mecanismo de selección y escritura de noticias, como un valor noticia más que interactúa con los tradicionales. Cuando Walter Lippman introdujo el concepto de valor noticia en los años 20 del pasado siglo, trataba de explicar por qué los periodistas cubrían algunos sucesos y pasaban por alto otros (Kepplinger, Hans Mathias; Ahmig, Simon Christine 2006: 25). Teóricos de la relevancia de Gonzalo. Martín Vivaldi, Lorenzo Gomis, José Luis Martínez Albertos y Josep María Casasús partieron de la clasificación de Warren para la valoración de los criterios de selección de la información (Casals, 2005: 195). En el 
ámbito internacional existe una larga tradición de estudio de los valores noticia que deben adaptarse a cada realidad y se reconfigura por una pragmática informativa que la activa y le otorga significado como puede ser el caso de la violencia (Garcia Orosa, Gallur, 2013). Consideramos que pese al incremento de factores y a la inclusión de nuevos agentes como las audiencias (Eilders, 2006: 9), continúan siendo válidos para la comprensión de mecanismos de construcción de realidades en los medios.

Dentro de esta corriente, partimos de la idea de estereotipo como imagen comúnmente aceptada inmutable conseguida a través de un conjunto de ideas, normas o patrones culturales previamente establecidos (Mateos de Cabo, 2007; Gerber, 2003; García Muñoz y Martínez García, 2008; Paniagua; Rossell, 2011). Así, estereotipar implicaría identificar al elemento estereotipado como el seguimiento de un modelo preestablecido, conocido y formalizado que se adopta de una manera permanente (Bach Arús et al., 2000: 44).

\section{Metodología}

El objetivo principal es el análisis del uso de los estereotipos de género como valor noticia en los diarios europeos. Como vimos, los estudios de género y medios de comunicación han demostrado la menor presencia de mujeres en el ámbito de la producción y los estereotipos incluidos en el discurso. En esta investigación se trató de demostrar que el estereotipo entra también en la fase intermedia de la producción de la información, la selección de información. La utilización de este valor noticia impide el acceso de muchas informaciones y promociona otras constituyendo no un elemento que el periodista incluye en el discurso sino que forma parte intrínseca del proceso de selección de noticias. Los acontecimientos son transformados a partir de categorías y modos de saber que constituyen la racionalidad periodística. El acontecimiento no es un dato, sino un constructo histórico-social dinámico e inestable $\mathrm{y}$, por lo tanto, al producir una noticia, el periodismo opera una lectura, un encuadre del mundo que produce su visibilidad, o sea, hace emerger el acontecimiento como información (Souza, Antunes, Ferreira, 2012: 388).

La investigación se desarrolló en dos fases:

a) Análisis cualitativo de los textos para inferir los estereotipos utilizados. Durante esta revisión, se detecta la existencia de "contra-estereotipos", se elabora una taxonomía de los mismos y se aplica a la muestra para poder comprobar su validez. En esta fase fueron revisadas 50.426 noticias en El Pais; 228.472 en Le Monde, 103.444 en The Times, 24.837 en El Corrieri della Sera; 33,062 en El Jornal.

Los estereotipos detectados se resumieron en el siguiente listado (en las tablas de la discusión de resultados se indicarán únicamente las siglas):

1. Madre (E.A.)

2. Débil (E.B)

3. Padece situaciones injustas para una mujer ya que se la ve como más débil que el hombre (E.C.)

4. Es una víctima, necesita ser protegida (E.D.)

5. Esposa o mujer de algún hombre "importante" o conocido (E.E.)

6. Debe ser perfecta (E.F.) 
7. Es guapa o debe serlo, cuando menos intentarlo (E.G.)

8. Recibe ayuda y tratamiento positivo por su condición de mujer (E.H.)

9. Realiza trabajos menos cualificados que los hombres (E.I.)

10. Mujer objeto (sufre agresiones sexuales por parte de los hombres) (E.K)

11. Es buena y lleva a cabo acciones positivas (E.L)

12. Debido a lo anterior cualquier acción negativa queda peor por el hecho de ser mujer (E.M).

13. Las mujeres embarazadas son difíciles de aguantar (E.N)

14. Heterosexual (E.Ñ.)

15. Hombres y mujeres son iguales en derechos, deberes, salarios y condiciones. (E.O.)

16. Es inferior y menos capaz que el hombre (E.P.)

La relación de "contra-estereotipos" elaborada fue la siguiente:

1. Mujer triunfadora que consigue llegar a dónde muy pocas llegan y situarse en trabajos de "hombres" (C.A.)

2. Quiere realizar actividades por sí misma (C.B.)

3. Quiere decidir por sí misma (C.C.)

4. Escala puestos en la sociedad en el sector económico, político y cultural (C.D.)

5. Es respetada (C.E.)

6. No tiene que ser protegida ya que no es víctima, debe autoprotegerse (C.F.)

7. Consigue alcanzar los mismos derechos que los hombres (C.G.)

8. Lucha por sus derechos (C.H.)

9. Los hombres reciben tratamiento de favor sobre las mujeres (C.I.)

b) Análisis de contenido comparativo de los cinco diarios europeos de los estereotipos y "contra-estereotipos" utilizados. Se analizaron todas las noticias con presencia femenina -1688 en total- publicadas desde 1 de mayo de 2013 hasta 1 de mayo de 2014.

Se seleccionaron medios de los cinco países incluidos en el estudio que estuvieran producidos por una empresa periodística de referencia en Europa que hubiese publicado un periódico en papel y, posteriormente, trasladara el medio a la red. Se eligieron el diario El Pais, de España; The Times, de Gran Bretaña; Le Monde, de Francia; Diario de Noticias, de Portugal y Corriere della Sera, de Italia. La unidad de análisis fue la noticia y el estudio se realizó sobre el hecho periodístico con el fin de comprobar la existencia de un valor noticia marcado por el estereotipo.

La hipótesis principal de la que partimos fue la siguiente: el concepto mujer se acaba convirtiendo en un valor noticia más en los medios de comunicación europeos. Así, en muchas noticias las mujeres salen en los medios porque el hecho, suceso o acontecimiento rompe o coincide con los estereotipos machistas que existen tradicionalmente.

A partir de la principal se elaboraron las siguientes hipótesis:

- El empleo de estereotipos o "contra-estereotipos" y la imagen creada de la mujer depende del medio de comunicación en el que se edite la misma. 
- En un número importante de casos las noticias sobre mujeres se refieren a situaciones y problemas típicamente "femeninos" según los estereotipos tradicionales tales como la maternidad, el parto, el aborto, la prostitución, la trata de blancas, la moda, la belleza, cuestiones domésticas, etc.

\section{Análisis de datos}

Después del estudio llevado a cabo en todas las noticias sobre mujeres en los medios digitales europeos desde el 1 de mayo del 2013 al 1 de mayo del 2014, se pueden establecer una serie de reflexiones que sintetizan y resumen los principales resultados que arroja esta investigación sobre los estereotipos usados en los medios.

En primer lugar, los estereotipos tradicionales sobre las mujeres (madre, débil, víctima, esposa de alguien, mujer objeto, etc.) están presentes en los cinco medios europeos, aunque en distinta medida y con diferente tratamiento dependiendo del periódico analizado. El estereotipo no figura como un elemento complementario o secundario de la noticia sino que está a lo largo de todo el texto y es destacado en el titular. De hecho, el estereotipo suele estar incluido ya en el hecho "noticiable", no en la "realidad" sino en el fragmento de la misma que el periodista observa y detecta como posible noticia. Así, actúa como un valor noticia más que adjunta a ese acontecimiento una importancia superior a la que tendría si el protagonista fuera un hombre y consigue que se transforme en noticia y figure en las páginas de los medios digitales. De este modo, encontramos titulares similares a los siguientes: "Cuando entramos en la Guardia Civil, no había números de botas para mujeres" (El País, 8 mayo de 2014) o "Las mujeres quieren decidir" (El País, 11 de abril de 2014), qué difícilmente podemos imaginar si sustituimos la palabra mujer por la palabra hombre. Sirvan como ejemplo otros titulares del medio francés: "Dirigeants, transmettez votre entreprise à une femme!" (Le Monde, 8 de mayo de 2014); "Jimmy Carter, l'homme à femmes" (Le magazine du Monde, 2 de mayo de 2014; "Last Days of Summer: quatre jours dans la vie d'une femme" (Le Monde, 29 de abril de 2014); "Le président de Clermont Foot: " Je ne prends pas plus de risques avec une femme comme entraîneur "” (Le Monde, 7 de mayo de 2014). Y The Times: "The woman, Be civilised and give presenting job to a woman, BBC urged" (The Times, 30 de abril de 2014) y "Woman joins referees'panel" (The Sunday Times, 11 de mayo 2014).

Los medios han incorporado no sólo a su discurso, sino también a sus rutinas productivas la imagen estereotipada de la mujer y esta característica del protagonista o protagonistas de los hechos podría formar parte de la estrategia de selección de hechos noticiables. La gravedad de la situación es incrementada, por lo tanto, por la dificultad de percepción por parte del receptor del uso del estereotipo más visible en el discurso que en el propio proceso de producción.

Pese a que en todos los medios analizados se detectó esta tendencia y, por ende, se registraron estereotipos en el discurso publicado por los mismos, existe una clara diferenciación en cuanto a la visión principal de las mujeres en El País, El Corriere della Sera y en el Jornal de Noticias, y la ofrecida en The Times y Le Monde (obsérvese tabla 1). En el primer grupo, destaca la presencia casi permanente de los principales estereotipos femeninos que presentan a la mujer fundamentalmente como víctima de su- 
cesos negativos, alguien que necesita protección. Así, accidentes, asesinatos y todo tipo de sucesos se presentan como más dramáticos todavía porque sus principales víctimas son mujeres, siendo claramente identificados dichos estereotipos como posibles valores-noticia que el medio aplica para considerar un hecho o acontecimiento relevante para la sociedad. Sirvan como ejemplo los siguientes titulares: "Ruba il telefonino a una donna, bloccato da due agenti fuori servicio" (Corriere della Sera, 12 de mayo de 2014); "Travolge una donna in motorino e scappa «Chi ha visto qualcosa chiami i vigili»»" (Corriere della Sera, 30 de abril de 2014), "Morde una donna all'orecchio per rapinarle la borsa" (Corriere della Sera, 19 de abril de 2014); "Sfonda le sbarre del passaggio a livello. Treno contro l'auto, donna gravissima" (Corriere della Sera, 17 de abril de 2014). Podemos encontrar múltiples ejemplos similares en El País en fechas de publicación muy próximas para ejemplificar su gran frecuencia: "La mujer de 80 años apuñalada en Alicante evoluciona favorablemente" (El País, 7 de abril de 2014); "Una mujer podrá hacer la oposición a la que no acudió por dar a luz" (El País, 1 de abril de 2014). Y, finalmente, en Jornal de Noticias: "Pena suspensa para mulher acusada de matar companheiro com uma garrafa de vino" (Jornal de Noticias, 24 de abril de 2014); "Mulher baleada em S. João da Pesqueira com "prognóstico favorável"" (Jornal de Noticias, 21 de abril de 2014); "Mulher caiu ao mar de altura de 35 metros e sobreviveu" (Jornal de Noticias, 20 de abril de 2014); "Mr. Bean troca mulher por atriz 28 anos mais nova" (Jornal de Noticias, 16 de abril de 2014).

Tabla 1. Estereotipos en los medios digitales europeos. Número de noticias.

Fuente: elaboración propia

\begin{tabular}{|c|c|c|c|c|c|c|c|c|c|c|}
\hline \multirow{3}{*}{$\begin{array}{c}\begin{array}{c}\text { Medio } \\
\text { analizado }\end{array} \\
\text { Estereotipos }\end{array}$} & \multirow{2}{*}{\multicolumn{2}{|c|}{$\begin{array}{l}\text { Jornal de noticias } \\
\text { Relación con el e. }\end{array}$}} & \multicolumn{2}{|c|}{ El País } & \multicolumn{2}{|c|}{ The Times } & \multicolumn{2}{|c|}{ Le Monde } & \multicolumn{2}{|c|}{$\begin{array}{c}\text { Corriere della } \\
\text { Sera }\end{array}$} \\
\hline & & & \multicolumn{2}{|c|}{ Relación con el e. } & \multicolumn{2}{|c|}{ Relación con el e. } & \multicolumn{2}{|c|}{ Relación con el e. } & \multicolumn{2}{|c|}{ Relación con el e. } \\
\hline & Continúa & Rompe & Continúa & Rompe & Continúa & Rompe & Continúa & Rompe & Continúa & Rompe \\
\hline E. A & 10 & 3 & 7 & 0 & 9 & 0 & 3 & 1 & 8 & 1 \\
\hline E. B & & 3 & 0 & 0 & 0 & 0 & 0 & 0 & 1 & 2 \\
\hline E. C & 1 & & 68 & 1 & 0 & 1 & 0 & 0 & 1 & 0 \\
\hline E. D & 167 & & 4 & 0 & 65 & 0 & 41 & 0 & 313 & 0 \\
\hline E. E & 13 & & 3 & 0 & 3 & 0 & 13 & 0 & 17 & 0 \\
\hline E. F & 3 & & 1 & 1 & 2 & 2 & 0 & 1 & 2 & 1 \\
\hline E. G & 11 & & 1 & 0 & 0 & 1 & 6 & 0 & 7 & 1 \\
\hline E. $\mathrm{H}$ & 17 & 2 & 11 & 1 & 14 & 1 & 0 & 0 & 1 & 0 \\
\hline E. I & & & 0 & 0 & 0 & 0 & 1 & 0 & 0 & 0 \\
\hline E. K & 5 & & 0 & 0 & 1 & 0 & 2 & 0 & 11 & 1 \\
\hline E. L & 2 & & 0 & 1 & 0 & 2 & 0 & 0 & 4 & 0 \\
\hline E. M & 55 & & 11 & 0 & 22 & 0 & 5 & 0 & 40 & 0 \\
\hline E. $N$ & & & 0 & 0 & 1 & 0 & 0 & 0 & 0 & 0 \\
\hline E. $\tilde{\mathrm{N}}$ & & 1 & 0 & 1 & 0 & 0 & 0 & 2 & 2 & 3 \\
\hline E. $\mathrm{O}$ & & 15 & 1 & 34 & 1 & 24 & 1 & 52 & 1 & 20 \\
\hline E. P & & & 1 & 0 & 0 & 1 & 0 & 0 & 1 & 0 \\
\hline
\end{tabular}


Como se puede observar en la tabla 1 existe una leve tendencia a la ruptura de esos estereotipos tradicionales con noticias que los trabajan negativamente pero el porcentaje todavía es muy pequeño en el grupo de los tres diarios comentados (poco más de 3 de cada 10 noticias).

Los estereotipos tradicionales están también presentes en The Times y Le Monde aunque en menor medida. Esto es fundamentalmente debido a que en ambos diarios aparecen tanto los estereotipos, como otra fórmula diametralmente opuesta a la anterior, que hemos denominado "contraestereotipo" (ver tabla 2), y que se caracteriza por presentar a la mujer con valores positivos (fuerte, triunfadora, decide por sí misma, independiente, lucha por sus derechos, realiza los mismos trabajos que el hombre, etc.), opuestos a los estereotipos machistas tradicionales. De este modo, el tono de una parte importante de las noticias deja de ser de sucesos para convertirse en relato de los logros de las mujeres. Así, mientras el tono de una parte de las noticias en $E l$ País, El Corriere della Sera y el Jornal de Notícias es derrotista y de cierto "menosprecio" a las capacidades de las mujeres, en The Times y Le Monde, los mismos titulares suelen mostrar una imagen positiva y triunfalista en noticias en las que las mujeres avanzan contundentemente hacia el logro de la igualdad con los hombres. Entre otros, podemos destacar como ejemplos los siguientes: "Dirigeants, transmettez votre entreprise à une femme!" (Le Monde, 8 de mayo de 2014); "Le président de Clermont Foot: « Je ne prends pas plus de risques avec une femme comme entraîneur "” ((Le Monde, 7 de mayo de 2014); "Municipales 2014: Anne Hidalgo devient la première femme maire de Paris" (Le Monde, 30 de marzo de 2014). Y en The Times: -"Kristin Scott Thomas on The Invisible Woman and her reunion with Ralph Fiennes" (The Times, 7 de febrero de 2014); "Inga Beale becomes first woman to lead Lloyds of London in its 325-year history" (The Times, 17 de diciembre de 2013); "Manifesto: the woman who runs the Crown's $£ 8$.6bn property jewels" (The Times, 8 de marzo de 2014); "Royal Society's first woman president warns of 'healing process' after poll" (The Times, 6 de febrero de 2014).

Tabla 2. Contra-estereotipos en los medios digitales europeos. Número de noticias.

Fuente: elaboración propia

\begin{tabular}{|l|c|c|c|c|c|c|c|c|c|c|}
\hline $\begin{array}{l}\text { Medio } \\
\text { analizado }\end{array}$ & \multicolumn{2}{|c|}{$\begin{array}{c}\text { Jornal de } \\
\text { noticias }\end{array}$} & \multicolumn{2}{|c|}{ El País } & \multicolumn{2}{c|}{ The Times } & \multicolumn{2}{c|}{ Le Monde } & \multicolumn{2}{c|}{$\begin{array}{c}\text { Corriere della } \\
\text { Sera }\end{array}$} \\
\hline $\begin{array}{l}\text { Contra- } \\
\text { estereotipo }\end{array}$ & Sólo & $\begin{array}{c}\text { Con } \\
\text { otros }\end{array}$ & Sólo & $\begin{array}{c}\text { Con } \\
\text { otros }\end{array}$ & Sólo & $\begin{array}{c}\text { Con } \\
\text { otros }\end{array}$ & Sólo & $\begin{array}{c}\text { Con } \\
\text { otros }\end{array}$ & Sólo & $\begin{array}{c}\text { Con } \\
\text { otros }\end{array}$ \\
\hline E. A & 7 & 0 & 14 & 0 & 15 & 0 & 11 & 0 & 18 & 0 \\
\hline E. B & 1 & 0 & 0 & 0 & 0 & 0 & 2 & 0 & 0 & 0 \\
\hline E. C & 3 & 0 & 4 & 0 & 2 & 0 & 0 & 0 & 4 & 0 \\
\hline E. D & 8 & 0 & 17 & 0 & 29 & 0 & 57 & 0 & 42 & 0 \\
\hline E. E & 5 & 0 & 9 & 0 & 11 & 0 & 17 & 0 & 8 & 0 \\
\hline E. F & 1 & 0 & 6 & 0 & 2 & 0 & 1 & 0 & 1 & 0 \\
\hline E. G & 1 & 0 & 3 & 0 & 0 & 0 & 1 & 0 & 1 & 0 \\
\hline E. H & 6 & 0 & 19 & 0 & 5 & 0 & 25 & 0 & 22 & 0 \\
\hline E. I & 3 & 0 & 2 & 77 & 0 & 0 & 0 & 0 & 1 & 0 \\
\hline
\end{tabular}


A pesar de todo lo anterior tanto un grupo de medios como el otro utilizan estereotipos, aunque de nuevo en el caso de usarlos los utilizan en grados y medidas distintas. Es decir, cuando The Times y Le Monde presentan noticias sobre sucesos negativos que les ocurren a las mujeres, el tono de la noticia es de indignación y de reclamo por el suceso ocurrido, mientras que esa misma noticia contada por El País, El Corriere della Sera y el Jornal de Notícias, es presentada en un tono de tragedia donde no se suele mostrar la indignación social o los reclamos de las propias mujeres, sino más bien se exponen datos. Esta tendencia va de la mano con lo que parece que es una concepción sobre las mujeres en los propios medios analizados. Así, por lo que ha sido investigado aquí se puede destacar que la "imagen" que proyectan los estereotipos femeninos utilizados en las noticias de El País, El Corriere della Sera o el Jornal de Notícias, es mucho más conservadora o tradicional, que la que se presenta en The Times y Le Monde. Estos últimos nos permiten conocer a una mujer desde una perspectiva mucho más liberal y moderna, sin victimismos y llena de energía.

Todo lo anterior se puede entender bien si hacemos hincapié en el hecho de que a pesar de que tanto estereotipos como "contra-estereotipos" están presentes en los cinco medios, la proporción es claramente distinta. Así, mientras en la mayor parte de las noticias de El País, El Corriere della Sera y el Jornal de Noticias se pueden identificar estereotipos, en una parte importante de lo publicado por The Times y Le Monde aparecen los "contra-estereotipos" o ideas positivas y "modernas" sobre la mujer. De modo más directo, los "contra-estereotipos" que hemos identificado en los medios analizados serían las rupturas que se hacen con respecto a las imágenes tradicionalmente aceptadas que supuestamente definían a una mujer, y por lo tanto, señalan ideas positivas que dan a entender que el sexo femenino ha entrado en una nueva era. Una era en la que las mujeres no necesitan pedir permiso para decidir y guiar su vida, para ser o no ser madres, para poder vivir su sexualidad con libertad, trabajar en los mismos puestos, igual de "duro" y cobrar lo mismo que el hombre.

Así, podemos señalar que en los medios europeos analizados se identifican "dos velocidades" distintas en el rechazo a la utilización de los estereotipos femeninos tradicionales (gráfico 1): aquellos medios que los han adaptado tan bien a sus rutinas productivas que ya forman parte de los valores noticia usados para decidir qué es y qué no es noticia (El País, El Corriere della Sera y el Jornal de Notícias) y aquellos otros que, a pesar de que todavía siguen usando estereotipos en algunas de las noticias publicadas, una parte importante de ellas tienen presente nuevas ideas positivas y modernas sobre las mujeres que cambian por completo una imagen sumisa, débil y sometida a la consideración de los hombres. Lo que sí queda claro es que los estereotipos sobre las mujeres están presentes en los medios de comunicación europeos, y a pesar de que su presencia es distinta dependiendo del país y del medio, su utilización está tan extendida que forma parte de la estrategia mediante la cual algunos medios seleccionan las noticias. 
Gráfico 1. Comparación de los estereotipos y estereotipos en los cinco periódicos. Porcentajes. Fuente: elaboración propia

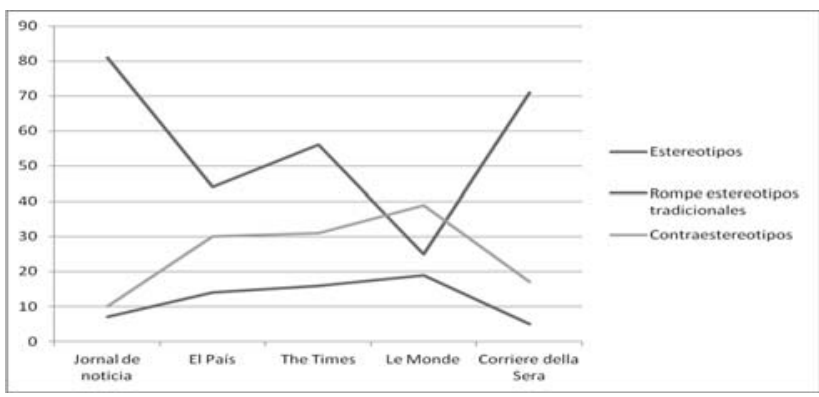

Podemos observar en las líneas superiores, una importante presencia de "contra-estereotipos" en Le Monde, The Times y El País, así como una frecuencia mucho más alta de estereotipos en Jornal de Noticias, El Corriere della Sera y The Times. El caso de este último medio llama especialmente la atención puesto que no sólo es el tercero en porcentajes de "contra-estereotipos", sino que además de ser el segundo en porcentaje de ruptura de estereotipos es igualmente el segundo en tanto por ciento de estereotipos.

\section{Conclusiones}

Los estereotipos siguen formando parte importante no sólo del discurso sino también del sistema de producción de la información en los medios digitales europeos. Sin embargo, en la red se detectan algunas tendencias -todavía minoritarias- pero esperanzadoras en este ámbito. La primera, es la ruptura de los patrones o imágenes tradicionales sobre la mujer $y$, la segunda, la figura de lo que denominamos "contra-estereotipos". No obstante, no existe un patrón único que nos permita analizar todos los medios, sino que cada uno marca una estrategia determinada que resumimos a continuación.

En primer lugar, puede apreciarse en este análisis dos tendencias diferenciadas y quizás una transición entre ambas. Le Monde se sitúa como el diario europeo de los analizados en los que aumenta en mayor medida en número de "contra-estereotipos" con respecto a los estereotipos, que disminuye sobremanera (en comparación al resto de medios). También es importante destacar que es el diario que más veces rompe el estereotipo de que hombres y mujeres estamos condiciones de igualdad, ofreciendo la imagen contraria. Es a la vez, el medio en el que más veces se muestra que las mujeres escalan puestos en la sociedad, que son respetadas y que luchan por sus derechos. Esto pudiera parecer algo sin importancia, sin embargo, las tendencias identificadas señalan que precisamente los medios donde más "contra-estereotipos" hay son aquellos donde se encuentran menos estereotipos, y a la vez son los mismos donde se rompen en más ocasiones con el estereotipo de que hombres y mujeres son iguales.

En la misma tendencia que Le Monde se sitúa The Times, con menos cantidades en cada uno de los rubros mencionados en "contra-estereotipos", pero con el mismo patrón: disminuye la cantidad de estereotipos con respecto al resto de los diarios europeos analizados, a la vez que aumenta el número de veces que se muestra que hombres 
y mujeres no están en condiciones de igualdad, y el de "contra-estereotipos". La tendencia intermedia de transición la muestra el diario El País, donde lo que más destaca es la atención prestada a la lucha de las mujeres por sus derechos. A la vez empieza a verse un mayor aumento del número de estereotipos sobre el de "contra-estereotipos".

Por último, justo en el extremo opuesto de la tendencia mostrada con Le Monde y The Times, tenemos al Jornal de Notícias, pero sobre todo a El Corriere della Sera. En el Jornal de Notícias se ve claramente como existen muy pocos "contra-estereotipos", siendo abrumadora la cantidad de estereotipos presentada al destacar la imagen de las mujeres como víctimas que necesitan se protegidas en 163 titulares. A pesar de la cifra anterior, el caso más extremo de todos es el de El Corriere de la Sera donde, debido a que hay más de 500 titulares con la palabra mujer en ellos las cifras son altas en general. Aún así, se aprecia lo exagerado de la tendencia al presentar dicho diario a la mujer como víctima que necesita ser protegida en 313 titulares, mientras en proporción el número de "contra-estereotipos" es de los más pequeños, al igual que el de veces que se rompe el estereotipo de que hombres y mujeres tienen igualdad de condiciones.

De todo lo anterior podemos destacar un llamativo patrón geográfico donde el diario italiano y el portugués, más al sur, son los que usan más estereotipos femeninos y menos "contra-estereotipos". El diario español se muestra a medio camino hacia una idea más progresista de la mujer, mientras que el diario inglés, pero sobre todo el francés se convierten en las cabeceras donde más se rompen los estereotipos "machistas" tradicionales sobre las mujeres, a la vez que se presentan a las mujeres a través de "contra-estereotipos" que refuerzan una imagen independiente y luchadora que puede desarrollar y desarrolla los mismos trabajos que los hombres. En ellos, además, destaca el hecho de que la mujer deja atrás su imagen de víctima que necesita ser protegida, o la de mujer de alguien, para decidir por sí misma, luchar por sus derechos y conseguir "escalar" posiciones en la sociedad.

A pesar de todo lo comentado previamente, quizás el caso más interesante es el del diario inglés donde podemos apreciar un número de estereotipos ligeramente mayor en número que el español, aunque ampliamente mayor en porcentaje (debido al menor número de noticias publicadas con la palabra mujer). Así podemos apreciar en The Times como la tendencia de aparición de ruptura de estereotipos y de utilización de "contra-estereotipos" en un número importante no es incompatible con el uso de ciertas formas de estereotipos, como serían: Mujer como madre, mujer como víctima, mujer objeto, acciones negativas peor vistas por el hecho de ser mujer. Si bien a la vez, el diario inglés deja claro una clara ruptura con la idea preconcebida de que hombres y mujeres somos iguales, señalando así una situación de evidente desigualdad sufrida por las mujeres. Con todo lo anterior se puede decir que en esta investigación ha aparecido un marcado patrón norte-sur en cuanto a la utilización de contra-estereotipos y la ruptura de las ideas tradicionales que se tienen sobre las mujeres, donde los diarios del norte de Europa usan más frecuentemente nuevas ideas no discriminatorias hacia las y rompen con las establecidas. Los del sur de Europa muestran una perspectiva más conservadora en cuanto a la utilización de ideas tradicionales preconcebidas sobre las mujeres. The Times aparece como un caso excepcional debido a que en el conviven el uso de "contra-estereotipos" y la ruptura de estereotipos con la utilización de estereo- 
tipos, presentándose así como un caso llamativo dentro de un patrón definido. El País se sitúa como punto intermedio de transición entre una tendencia y otra.

\section{Referencias bibliográficas}

ALANDRO VICO, Eva; PADILLA CASTILLO, Graciela; REQUEIJO REY, Paula; SEMOVA, Dimitrina Jivkova; GARCÍA AGUSTÍN, Julia; GARCÍA NIETO, María Teresa; y VIÑARÁS ABAD, Mónica (2014): "La presencia y representación de la mujer científica en la prensa española". Revista Latina de Comunicación Social, 69, pp. 176-194. DOI: 10.4185/RLCS-2014-1007

BACH, Marta; ALTÉS, Elvira; GALLEGO, Juana; PLUJA, Marta; y PUIG, Montserrat, (2000): El sexo de la noticia. Barcelona, Icaria Editorial.

CASALS CARRO, María Jesús (2005): Periodismo y sentido de la realidad. Teoría $y$ análisis de la narrativa periodística. Madrid, Fragua.

EILDERS, Christiane (2006): "New factors and news decisions. Theoetical and Methodological advances in Germany". Comunications: The European Journal of Communication Research, 31, 1, pp. 5-24.

FISHMAN, Mark (1980): Manufacturing the news: the Social Organitation of Media News Production. Santa Bárbara, Universidad de California.

FRANQUET CALVET, Rosa; LUZÓN FERNÁNDEZ, Virginia; y RAMAJO, Natividad (2007): "La información en los principales medios de comunicación on line. Estudiar la representación de género". Zer. Revista de estudios de comunicación, 22, pp. 267-282.

GALLEGO, Juana (2013): De reinas a ciudadanas. Medios de comunicación, ¿motor o rémora para la igualdad?. Barcelona, Aresta.

GALLEGO, Juana (dir., 2002): La prensa por dentro. Producción informativa y transmisión de estereotipos de género. Barcelona, Los libros de la frontera.

GARCÍA MUÑOZ, Nuria y MARTÍNEZ GARCÍA, Luisa (2008): "La recepción de la imagen de las mujeres en los medios: una aproximación cualitativa". Nueva Época, 10, julio-diciembre, pp. 111-128.

GARCÍA MUÑOZ, Nuria y MARTÍNEZ, Luisa (2009): “La representación positive de la imagen de las mujeres en los medios", Comunicar, 32, XVI, pp. 209-214.

GARCÍA OROSA, Berta y GALLUR SANTORUM, Santiago (2012): "La conformación de valores noticia en un contexto de conflicto. Análisis pragmático de su funcionamiento en la información sobre el narcotráfico en México y el feminicidio de Ciudad Juárez". Estudios sobre el Mensaje Periodístico, vol. 18, núm. 2, pp. 491-511. Madrid, Servicio de Publicaciones de la Editorial Complutense.

GERBER, Elisabet (coord., 2003): Género y comunicación. Las mujeres en los medios masivos y en la agenda política. Buenos Aires, Fundación Friedrich Ebert en la Argentina.

GÓMEZ Y PATIÑO, María (2011): “Análisis del tratamiento de la mujer en la prensa española. Día Internacional de las Mujeres”. Estudios sobre el Mensaje Periodís- 
tico, vol. 17, núm. 1, pp. 119-140. Madrid, Servicio de Publicaciones de la Universidad Complutense.

KEPPLINGER, Hans Mathias and AHMIG, Simon Christine (2006): "Predicting news decisión. An empirical test of the two component theory of news selection". Comunications: The European Journal Of Communication Research, 31, num. 1, pp. 25-40.

KIVIKURU, Ullamaija (1999): Images of Women in the Media. Bruxelles, European Commission.

MARÍN, Flora; ARMENTIA, Jose Ignacio; y CAMINOS, José (2011): "El tratamiento informativo de las víctimas de violencia de género en Euskadi: Deia, El Correo, El País y Gara (2002-2009)". Comunicación y Sociedad, 24, núm. 2, pp. 435-466.

MARÍN, Flora y GANZABAL, María (2011):” La mujer (in)visible: la construcción de la identidad femenina a través de la fotografía en El País y El Mundo".Enl@ce Revista Venezolana de Información, Tecnología y Conocimiento, 8, 3, pp. 51-67.

MATEOS DE CABO, Ruth; GIMENO NOGUÉS, Ricardo; y MARTÍNEZ MARTÍNEZ, Miryam (2009): "Presencia de estereotipos en los medios de comunicación: análisis de la prensa digital española", en COSSIO-SILVA, Francisco José: Administrando en entornos inciertos. Madrid, Escuela Superior de Gestión Comercial y Marketing, ESIC.

PANIAGUA, Bernardo; PELLISER ROSELL, Ne-lo; y AGUILAR SOLVES, Teresa (2011): "La construcción de estereotipos de género en Canal 9. Un estudio crítico de "l'Alquería Blanca". Actas del II Congreso CONGENERE "La Construcció de gènere a la ficció televisiva". Girona, Universitat de Girona.

SOUZA LEAL, Bruno; ANTUNES, Elton; y FERREIRA VAZ, Paulo (2012): "E1 acontecimiento como contenido de las noticias: repensando una metodología". Estudios sobre el Mensaje Periodístico, vol. 18, núm. 1, pp. 383-398. Madrid, Servicio de Publicaciones de la Universidad Complutense.

TOUS-ROVIROSA, Ana; MESO AYERDI, Koldo; y SIMELIO SOLA, Nuria (2013): "The Representation of Women's Roles in Television Series in Spain. Analysis of the Basque and Catalan Cases". Comunicación y Sociedad, 26, 3, 2013, pp. 67-97.

TUCHMAN, Gaye (1983): La producción de la noticia. Estudio sobre la construcción de la realidad. México, Gustavo Gili.

Santiago Gallur Santorum es Profesor titular de la Universidad Autónoma de Ciudad Juárez (Chihuahua, México)

Berta García Orosa es Profesora titular de la Facultad de Ciencias de la Comunicación de la Universidad de Santiago de Compostela 